\title{
Dasatinib synergises with irinotecan to suppress hepatocellular carcinoma via inhibiting the protein synthesis of PLK1
}

Li Xu ${ }^{1,2}$, Yuanrun Zhu ${ }^{1,2}$, Jinjin Shao ${ }^{1}$, Min Chen ${ }^{1}$, Hao Yan ${ }^{1}$, Guanqun $\mathrm{Li}^{1}$, Yi Zhu ${ }^{1}$, Zhifei Xu ${ }^{1}$, Bo Yang ${ }^{1}$, Peihua Luo*,1 and Qiaojun $\mathrm{He}^{\star, 1}$

'Zhejiang Province Key Laboratory of Anti-Cancer Drug Research, Institute of Pharmacology and Toxicology, College of Pharmaceutical Sciences, Zhejiang University, Hangzhou 310058, China

Background: Hepatocellular carcinoma $(\mathrm{HCC})$ is one of the most common types of malignant tumour and has poor prognosis. Currently, systematic chemotherapy is the only approach to prolong survival. Thus the development of new treatment regimens is urgently needed to improve the therapeutic efficacy. Our study intended to assess the combination of dasatinib and irinotecan against HCC and made an effort to develop a potential medical choice for advanced HCC patients.

Methods: We used SRB colorimetric assay and clonogenic assay to assess antitumour effect in vitro and HCC xenograft model to assess antitumour effect in vivo. We applied flow cytometry and western blotting to explore the mechanism of the combined therapy. Knockdown and overexpression of PLK1 are also applied for validation.

Results: We confirmed that dasatinib has synergistic effect with irinotecan (or SN38) on HCC both in vitro and in vivo. The effect is due to arisen apoptosis rate of $\mathrm{HCC}$ cells that is accompanied by mitochondria dysfunction. The enhanced antitumour efficacy of SN38 could be explained by additional inhibition of PLK1, which is triggered by dasatinib. Unlike existed PLK1 inhibitors, dasatinib does not inhibit PLK1 activity in a direct way. Instead, we found that dasatinib reduces PLK1 level by interfering with its protein synthesis progress. We validated that this kind of downregulation of PLK1 level has a key role in the synergistic effect of the two agents.

Conclusions: Dasatinib is able to reinforce the anti-HCC efficacy of irinotecan/SN38 by downregulation of PLK1 synthesis. The combination of the two agents might be a potential medical choice for HCC therapy.

As one of the most common cancers, hepatocellular carcinoma (HCC) is the second cause of death by cancer and the overall median survival for HCC patients is $<6$ months currently ( $95 \%$ CI: 5.9-6.1; Njei et al, 2015; Torre et al, 2015). Owing to the difficulty in accurate and reliable diagnosis of early-stage HCC, most patients are confirmed as advanced stage (Bruix et al, 2011). For advanced HCC, the only first-line drug is sorafenib, which unfortunately has a limited effect on HCC. Therefore, a novel and effective clinical treatment strategy for HCC is urgently needed (Bupathi et al, 2015).
Irinotecan, a kind of DNA Topoisomerase-I inhibitor, has been widely used as a first-line chemotherapy in some solid malignant tumours, such as colon cancer (Ottolino-Perry et al, 2015). Numerous studies (Takeba et al, 2007) showed that SN38 (the in vivo active metabolite of irinotecan) had superior inhibition ability against HCC in vitro, whereas the phase II studies of irinotecan on HCC failed owing to its dose-dependent toxicity (O'Reilly et al, 2001). Consequently, a combination therapy with lower dose to decrease the toxicity of irinotecan will be a potential strategy for its application on HCC.

*Correspondence: Professor P Luo; E-mail: peihualuo@zju.edu.cn or Professor Q He; E-mail: qiaojunhe@zju.edu.cn

${ }^{2}$ These authors contributed equally to this work. 
Dasatinib is a kind of tyrosine kinase inhibitor (TKI) targeted on $\mathrm{Abl}, \mathrm{c}$-kit and Src (Montero et al, 2011) and has been used to treat patients with Philadelphia chromosome-positive leukaemia (Talpaz et al, 2006)and several kinds of solid tumours (Mayer et al, 2011). It has been reported that dasatinib combined with traditional chemotherapeutic agents could reveal additional antitumour effect (Milano et al, 2009; Secord et al, 2012; Somlo et al, 2013; Viktorsson et al, 2016). The approaches in which dasatinib shows these effects are various. One of the mechanisms is decreasing protein biosynthesis through the modulation of target genes (Nunoda et al, 2007).

As is well known, the overexpression caused by increased protein biosynthesis is one of the ways in which oncogenes are activated. In other words, the reduction of synthesis is a possible method to inhibit oncogenes. Polo-like kinase 1 (PLK1) is a wellstudied regulator in mitosis and has key role in numerous mitotic events (Barr et al, 2004). It also directly takes part in DNA integrity maintenance and regulation of DNA repair according to accumulating evidence (Chabalier-Taste et al, 2016). Of note, PLK1 is overexpressed in HCC (He et al, 2009) and has been proven to be one of the factors that could determine SN38 sensitivity of tumour cells (Zuco et al, 2015). These facts indicate that the inhibition of PLK1 protein biosynthesis progress may confront the overexpression of PLK1 and consequently enhance the effect of SN38 on HCC. Here we hypothesised that dasatinib could interfere with PLK1 synthesis and show synergistic antitumour effect with SN38.

In this study, we confirmed that the combination of irinotecan and dasatinib has synergistic antitumour effect on HCC and exhibited that the synergistic effect was a result of inhibition of PLK1 through the downregulation of its synthesis and consequently induced increasing apoptosis of cancer cells. Therefore, the combination use of irinotecan and dasatinib might be an effective strategy for clinical therapy of HCC.

\section{MATERIALS AND METHODS}

Cell lines and cell culture. HepG-2 and BEL-7402, the human hepatocellular carcinoma cell lines, were purchased from Cell Bank of China Science (Shanghai, China). And cells were maintained in DMEM/RPMI-1640 containing 10\% heat-inactivated fetal bovine serum (FBS) and $0.1 \%$ antibiotics at $37^{\circ} \mathrm{C}$ in a humidified atmosphere with $5 \% \mathrm{CO}_{2}$.

Drugs and reagents. Dasatinib was obtained from the first Affiliated Hospital, Zhejiang University School of Medicine, Hangzhou, China. Irinotecan and SN38 were kindly provided by Dr Wei Lu (East China Normal University). Primary antibodies against GAPDH and ubiquitin HRP-labelled secondary antibodies were purchased from Santa Cruz Biotechnology (Dallas, TX, USA). Primary antibodies against cleaved PARP, cleaved caspase- 3 and PLK1 were obtained from Cell Signaling Technology (Beverly, MA, USA). DMSO, sulphorhodamine B (SRB), Trichloroacetic acid, Tris base, propidium iodide (PI) and DAPI (4', 6-diamidino-2phenylindole) were obtained from Sigma-Aldrich (St Louis, MO, USA).

Cell proliferation assays. SRB colorimetric assay was applied to analyse cell proliferation (Vichai and Kirtikara, 2006). Cells were plated in 96-well plates at a density of 4000 cells per well and left for $24 \mathrm{~h}$ growth in $5 \% \mathrm{CO}_{2}$ incubator at $37^{\circ} \mathrm{C}$. After exposed to different concentrations of dasatinib, SN38 or dasatinib combined with SN38 for $48 \mathrm{~h}$, cells were fixed with $10 \%$ (wt/vol) trichloroacetic acid and stained with SRB for $30 \mathrm{~min}$. SRB was dissolved in $10 \mathrm{mmoll}^{-1}$ Tris base for absorbance measurement using Multiskan Spectrum (Thermo Electron Corporation, Marietta, $\mathrm{OH}, \mathrm{USA}$ ) at $510 \mathrm{~nm}$. And the inhibition rate of cell proliferation was calculated for each well as ((the absorbance of control cells - the absorbance of treated cells)/the absorbance of control cells) $\times 100 \%$.

Combination index (CI). Based on the results of SRB colorimetric assay, CI value was calculated according to the Chou-Talalay method (Chou, 2010) using the CalcuSyn software (version 2; Biosoft, Cambridge, UK). It indicates synergistic, summation and antagonistic interaction between two drugs when CI values are $<1.0,=1.0$ and $>1.0$.

Colony-formation assay. HepG-2 or BEL-7402 cells were plated at 1000 per well in a six-well plate and treated with dasatinib and/ or SN38. Then, every 3 days, medium with 10\% FBS were replaced and drugs were re-added. After 9 days of treatment, the cells were stained with a $0.1 \%$ crystal violet solution and photographed. The crystal violet was dissolved in $10 \%$ acetic acid and the absorbance values were detected at $595 \mathrm{~nm}$.

Crystal violet was dissolved in $10 \%$ acetic acid and the absorbance values were determined at $595 \mathrm{~nm}$. The clonogenic ability was calculated as ((the absorbance of treated cells - the absorbance of blank)/(the absorbance of control cells - the absorbance of blank) $) \times 100 \%$.

Flow cytometric assay. Flow cytometric assay was applied to detect apoptosis by Annexin V-FITC/PI apoptosis kit. HepG-2 and BEL-7402 cells were treated with dasatinib and/or SN38 for $48 \mathrm{~h}$ and harvested. Then cells were resuspended in $500 \mu \mathrm{l}$ binding buffer and incubated with $5 \mu \mathrm{l}$ of Annexin V-FITC and $5 \mu \mathrm{l}$ of PI at room temperature for $15 \mathrm{~min}$. Samples were then analysed on a FACS Calibur cytometer (Becton Dickinson, Franklin Lakes, NJ, USA).

DAPI staining. In all, $2 \times 10^{5}$ per well HepG- 2 cells were cultured in a six-well plate and treated with dasatinib $\left(80 \mathrm{nmoll}^{-1}\right)$ and/or SN38 $\left(20 \mathrm{nmoll}^{-1}\right)$ for $48 \mathrm{~h}$. And then all the cells were fixed with $4 \%$ paraformaldehyde for $30 \mathrm{~min}$ and permeabilised with PBS containing $0.1 \%$ Triton X-100. Cells were washed with PBS and stained with DAPI solution $\left(1.0 \mu \mathrm{g} \mathrm{ml}^{-1}\right)$. Fluorescence was observed with Olympus Fluorview 1000 confocal microscope (Olympus Corporation, Tokyo, Japan).

Western blotting. HepG-2 and BEL-7402 cells were seeded in sixwell plates $\left(2 \times 10^{5}\right.$ per well) and treated with dasatinib and/or SN38 for $48 \mathrm{~h}$. After drug treatment, cells were harvested and resuspended in lysis buffer $\left(50 \mathrm{mmoll}{ }^{-1}\right.$ Tris- $\mathrm{HCl}, 150 \mathrm{mmoll}^{-1}$ $\mathrm{NaCl}, 2 \mathrm{mmoll}^{-1}$ EDTA, $2 \mathrm{mmoll}^{-1}$ EGTA, $25 \mathrm{mmoll}^{-1} \mathrm{NaF}$, $25 \mathrm{mmoll}^{-1} \quad \beta$-Sodium Glycerophosphate, $0.3 \%$ NP-40, $0.3 \%$ Triton X-100, 0.25\% Leupeptin, 0.1\% PMSF, 0.1\% $\mathrm{NaVO}_{3}$ ). Tumour tissue samples were sonicated in lysis buffer. All the samples were then centrifuged at 13200 r.p.m. at $4{ }^{\circ} \mathrm{C}$ for $30 \mathrm{~min}$. Protein samples were separated on Tris-glycine gels and transferred to nitrocellulose membrane and then incubated with corresponding HRP-linked antibody. Finally, protein bands were detected with the ECL-Plus Kit (PerkinElmer, MA, USA) and visualised on autoradiography film.

Real-time reverse transcription-PCR (qRT-PCR). HepG-2 cells $\left(2 \times 10^{5}\right.$ per well $)$ were seeded in a six-well plate and treated with dasatinib $\left(80 \mathrm{nmoll}^{-1}\right)$ and/or SN38 $\left(20 \mathrm{nmoll}^{-1}\right)$ for $48 \mathrm{~h}$. Then total RNA was extracted with TRIZOL (Invitrogen, Boston, MA, USA), and cDNA was prepared by reverse transcription of $2 \mu \mathrm{g}$ samples of total RNA using TransScript One-Step gDNA Removal and cDNA Synthesis SuperMix (Beijing Transgenbiotech, Beijing, China). And specific primers for PLK1 (Forward: 5'-TAATGACT CAACACGCCTGATT- $3^{\prime}$; Reverse: $5^{\prime}$-AGCTCAGCAGCTTGTC TACCAT- $3^{\prime}$ ) and GAPDH (Forward: $5^{\prime}$-GTCATCCATGACA ACTTTGG-3'; Reverse: $5^{\prime}$-GAGCTTGACAAAGTGGTCGT-3') were used to perform qRT-PCR. PCR was run on Applied 
Biosystems 7500 Fast Real-Time PCR System (Applied Biosystems, Woburn, MA, USA).

Plasmids transfection. PLK1 plasmids(SC110978) were purchased from Origene Technologies Inc (Rockville, MD, USA). HepG-2 cells were cultured in a six-well plate and PLK1 plasmids were transfected $24 \mathrm{~h}$ later using Lipofectamine 2000 (Invitrogen, 11668-019), according to the manufacturer's instructions.

siRNA transfection. PLK1 siRNA (\#1: 5'-GCACAUACCGCCU GAGUCU-3'; \#2: $5^{\prime}$-CCACCAAGGUUUUCGAUUG- ${ }^{\prime}$ ) and negative control siRNA (NC) were obtained from GenePharma Co. Ltd (Shanghai, China). HepG-2 cells $\left(2 \times 10^{5}\right.$ per well) were seeded in a six-well plate and, after $24 \mathrm{~h}$, siRNA were transfected with Oligofectamine2000 (Invitrogen, 12252-011), according to the manufacturer's instructions.

Detection of treatment efficacy in vivo. In all, $5 \times 10^{6} \mathrm{HepG}-2$ cells were injected subcutaneously to 24 female 5-6-week-old $\mathrm{BALB} / \mathrm{c}$ mice. All the mice were randomly divided into four groups (control, treated with dasatinib, treated with irinotecan, treated with both dasatinib and irinotecan) when the tumour volume reached about $100 \mathrm{~mm}^{3}$. Dasatinib $\left(100 \mathrm{mg} \mathrm{kg}^{-1}\right.$, dissolved in $0.5 \%$ $\mathrm{CMC}-\mathrm{Na})$ was applied once daily and/or irinotecan $\left(1 \mathrm{mg} \mathrm{kg}^{-1}\right.$, dissolved in physiologic saline) was applied every 2 days for 30 days. Tumour volume $(V)$ and body weight were measured every 2 days and the tumour volume $(V)$ was calculated as $V=($ length $\times$ width $\left.^{2}\right) / 2$. All animal experiments complied with the ARRIVE guidelines. The in vivo research carried out in the study titled 'Dasatinib synergises with irinotecan to suppress hepatocellular carcinoma via inhibiting the protein synthesis of PLK1' was approved by Center for Drug Safety Evaluation and Research of Zhejiang University. The project number is P-IACUC-14-002.

Statistical analyses. All data are presented as mean \pm s.d. Statistical significance were analysed by Student's $t$-test or oneway ANOVA analysis using SPSS 19.0 (IBM, New York, NY, USA) and differences were considered statistically significant when $P$ value $<0.05$.

\section{RESULTS}

Cytotoxicity of the combination of dasatinib and SN38 in human HCC cell lines. In our primary study, we assessed the antitumour effect of the combination of SN38 and dasatinib in human HCC cell lines, including HepG-2 and BEL-7402. Cells were treated with serial concentrations of SN38, dasatinib or both for $72 \mathrm{~h}$, and SRB staining assay was used to detect the survival fractions of each treatment group. The survival curves of SN38, dasatinib and combination are shown in Figure 1A. The survival infractions were $55.8 \pm 6.2 \%, 50.9 \pm 4.1 \%$ and $20.8 \pm 4.9 \%$, respectively, in HepG-2, when cells were exposed to SN38 (20 nM),
A
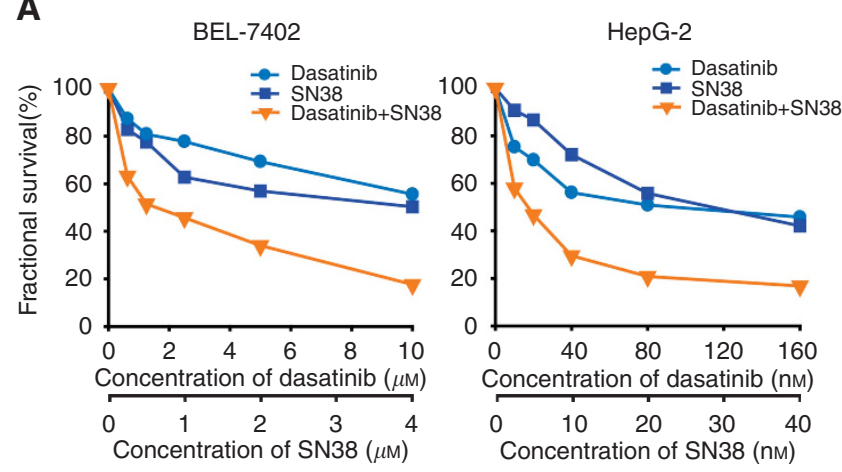

B

\begin{tabular}{cccccc}
\hline \multicolumn{3}{c}{ BEL-7402 } & \multicolumn{4}{c}{ HepG-2 } \\
\hline Dasa $(\mu \mathrm{M})$ & SN38 $(\mu \mathrm{M})$ & $\mathrm{Cl}$ & Dasa $(\mathrm{nm})$ & SN38 $(\mathrm{nm})$ & $\mathrm{Cl}$ \\
\hline 0.625 & 0.250 & 0.271 & 10.000 & 2.500 & 0.333 \\
1.250 & 0.500 & 0.241 & 20.000 & 5.000 & 0.323 \\
2.500 & 1.000 & 0.327 & 40.000 & 10.000 & 0.222 \\
5.000 & 2.000 & 0.286 & 80.000 & 20.000 & 0.242 \\
10.000 & 4.000 & 0.132 & 160.000 & 40.000 & 0.351 \\
\hline
\end{tabular}

C

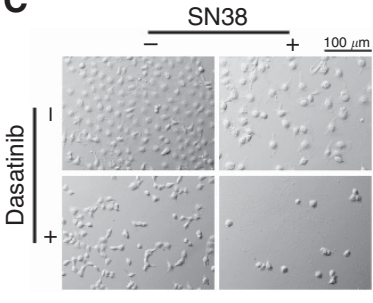

D

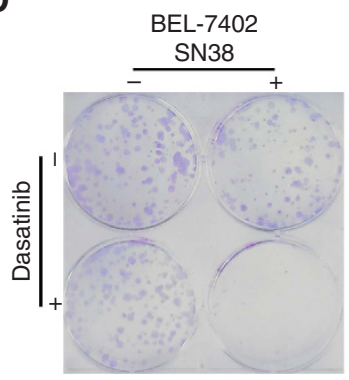

BEL-7402

E

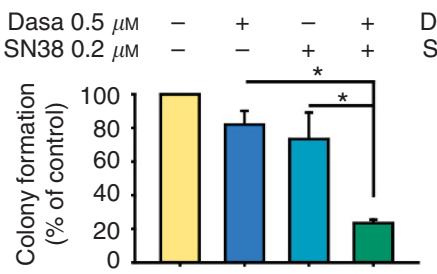

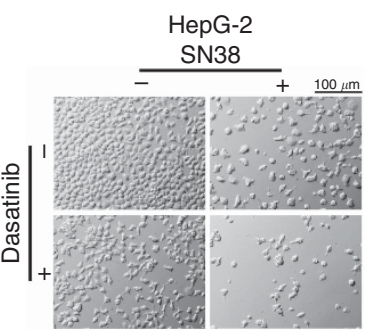

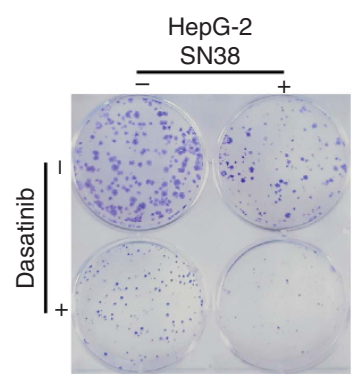

HepG-2

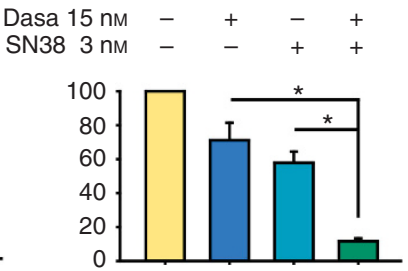

Figure 1. Combinational cytotoxicity of dasatinib and SN38. (A) Combination treatment of dasatinib and SN38 induced cytotoxicity in HCC cell lines, including BEL-7402 and HepG-2. And $4 \times 10^{3}$ cells per well were cultured in 96 -well plates and exposed to different concentrations of dasatinib and/or SN38 for $48 \mathrm{~h}$. Survival fractions were calculated and shown as mean \pm s.d. from three independent experiments. (B) $\mathrm{Cl}$ values at different ratio concentrations of dasatinib and SN38 were calculated by the proliferation inhibition rates. (C) The inhibition of cell proliferation was observed by optical microscopy after combination treatment for $48 \mathrm{~h}$ (magnification $\times 200$ ). (D) Colony-formation assays of BEL-7402 and HepG-2 were performed. One thousand cells were plated in a six-well plate and incubated with dasatinib and/or SN38 for 10 days and then photographed. (E) The colony-formation ability of each group was shown in bar chart and described as mean \pm s.d. from three independent experiments. ${ }^{\star}$ Compared with the combination group, $P<0.05$. 
dasatinib $(80 \mathrm{~nm})$ or combination. And those in BEL-7402 were $50.3 \pm 5.0 \%, \quad 55.6 \pm 2.4 \%$ and $17.6 \pm 4.9 \%$ respectively, when cells were exposed to SN38 $(4 \mu \mathrm{M})$, dasatinib $(10 \mu \mathrm{M})$ or combination, which suggested that the combination therapy between SN38 and dasatinib significantly reduced the survival fraction, compared with each agent alone. In order to verify the synergistic effect of dasatinib and SN38, CI values were calculated. As is shown in Figure 1B, the CI value of most combined concentration of dasatinib and SN38 are $<0.9$, which demonstrated the evident synergism of these two drugs on HepG-2 and BEL-7402.

To explore whether the combination treatment of SN38 and dasatinib can induce a long-term synergistic antitumour effect, we performed a clonogenic assay. HepG-2 and BEL-7402 cells were respectively treated with monotherapy or co-treatment for $24 \mathrm{~h}$, and then cells in each group were normally cultured for clone formation at the same count. The typical pictures after 20-day culture are shown in Figure 1D, in which combination treatment resulted in a significant inhibition to the proliferation of HepG-2 and BEL-7402 cell clone while the monotreatment induced a moderate inhibition. Simultaneously, we calculated the rates of colony formation compared with the control group. Consistent with the picture, the HepG-2 colony-formation rates treated by dasatinib, SN38 and combination were $71.2 \pm 10.3 \%$,
$57.9 \pm 6.6 \%$ and $11.1 \pm 1.7 \%$, respectively. And the BEL-7402 colony-formation rates treated by dasatinib, SN38 and combination were $82.0 \pm 8.1 \%, 73.4 \pm 15.7 \%$ and $23.4 \pm 2.1 \%$, respectively (Figure 1E), indicating that the combination treatment performed a long-term synergistic effect on the survival and proliferation of HCC cells. Taken together, combination of dasatinib and SN38 showed reinforced antitumour effect on human HCC cell lines in vitro.

The combination of $\mathrm{SN} 38$ and dasatinib results in enhanced caspase-dependent apoptosis in human HCC cell lines. In order to further confirm the synergistic antitumour effect, we then monitored the apoptosis of HepG-2 and BEL-7402 cells after 48-h exposure to SN38 (2 $\mu \mathrm{M}$ for BEL-7402 and $20 \mathrm{nM}$ for HepG-2), dasatinib ( $5 \mu \mathrm{m}$ for BEL-7402 and $80 \mathrm{~nm}$ for HepG-2) or both. The results detected by AV-PI staining using flow cytometry are shown in Figures $2 \mathrm{~A}$ and $\mathrm{B}$, in which the apoptosis rates of the control, dasatinib, SN38 and combination groups in HepG-2 were 18.20, $18.21,30.62$ and $44.35 \%$, respectively, and those in BEL-7402 were $29.07,30.09,41.94$ and $71.06 \%$, respectively. Those results suggested that the combination of two agents resulted in an enhanced apoptosis in HCC cells, compared with monotreatment. In order to visualise the apoptosis through nucleus morphology, DAPI staining for HepG-2 cells was used and the typical pictures
A
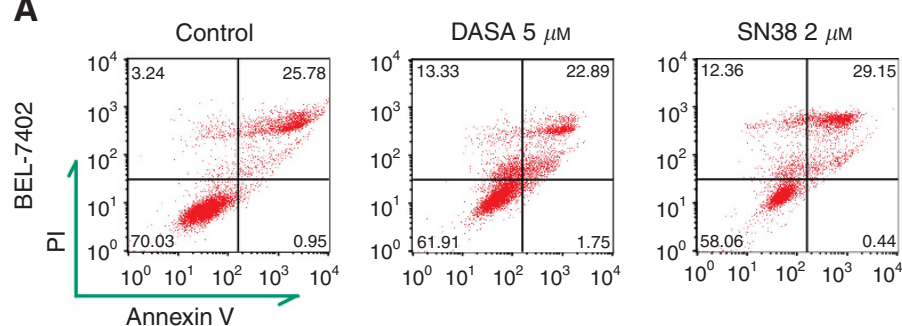

B
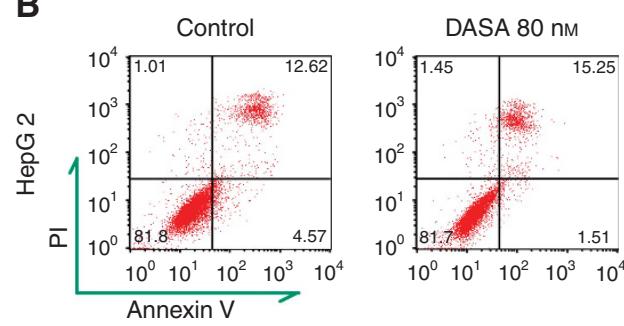

C

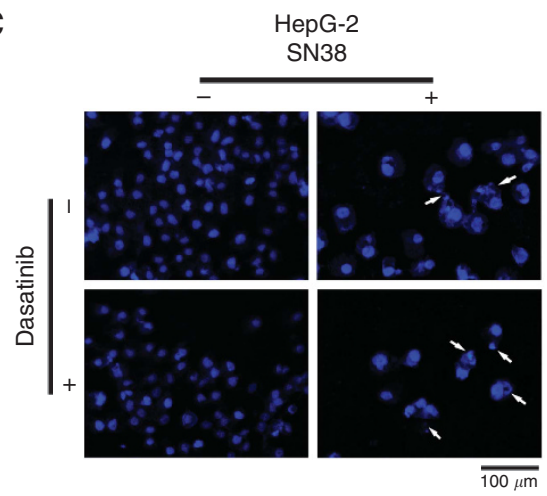

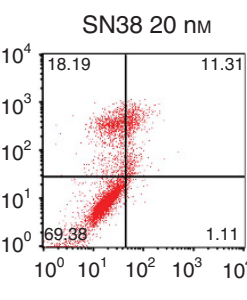

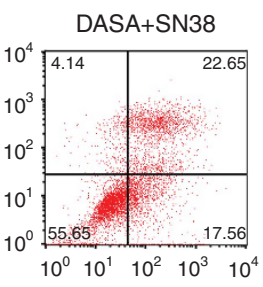

D

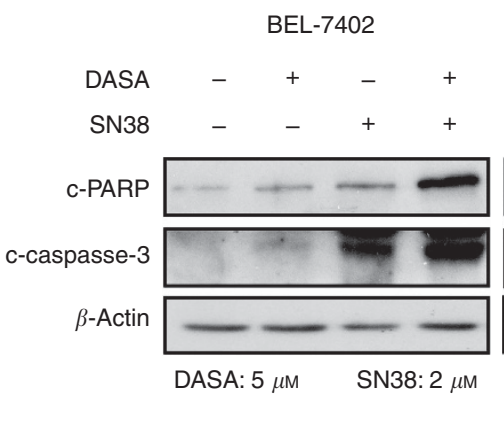

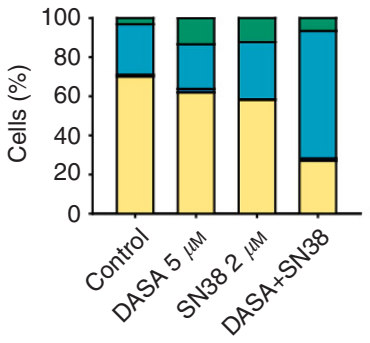
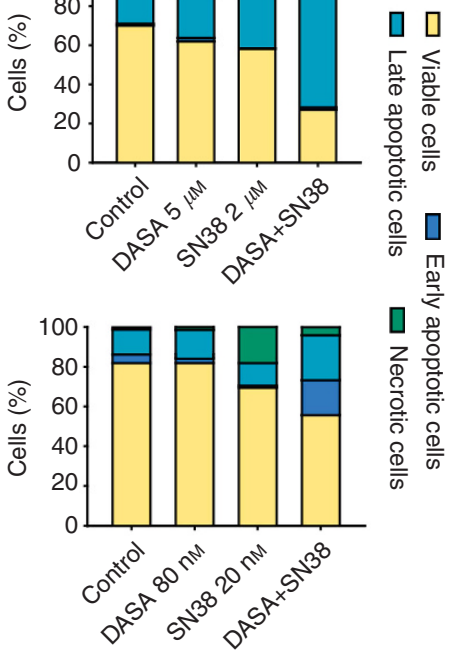

HepG-2

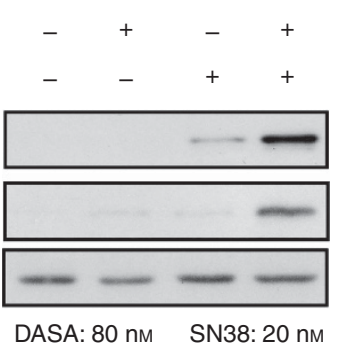

Figure 2. SN38-induced caspase-dependent apoptosis was enhanced by dasatinib in HCC cells. (A, B) Annexin V-PI staining assays were applied to detect apoptosis. After treatment with dasatinib and/or SN38 for 48 h, BEL-7402 and HepG-2 were harvested and stained with Annexin V and PI. Apoptosis rates were analysed by flow cytometry. Quality control results are accessible in Supplementary Figure S1. (C) Morphology of apoptosis is shown in HepG-2 cells treated with dasatinib, SN38 or combination for $48 \mathrm{~h}$. DAPI staining and fluorescence microscope were used to detect nuclear condensation and cellular fragmentation (magnification $\times 200$ ). (D) Western blotting proved the increased apoptosis in the combination treatment group. Proteins were extracted from cells of different groups, and specific antibodies were applied to detect cleaved PARP, cleaved caspase- 3 and $\beta$-Actin. 
are shown in Figure 2C. In the combination group, extensive karyopyknosis and cellular fragmentation were observed (as indicated by the white arrows), while other treatments failed to arise the nucleus morphology alteration, which suggested that higher rate of apoptosis existed in the combination group.

As caspase-dependent pathway is an important pathway involved in cell apoptosis, we monitored the activation of the caspase cascade in HCC cells after the treatment with dasatinib and/or SN38 by western blotting analysis. It is known that cleaved PARP and cleaved caspase- 3 represent occurrence of apoptosis (Jin and El-Deiry, 2005). We found that dasatinib or SN38 were able to increase the cleavage of PARP and caspase-3 slightly, and these effect can be remarkably enhanced when applied together, which indicated that apoptosis was triggered by caspase cascades (Figure 2D). In sum, the above results proved that the combination use of dasatinib and SN38 led to increased apoptosis through caspase cascades in HCC cells, which finally caused more cell death.

Mitochondrial dysfunction-dependent apoptosis was involved in the combination of SN38 and dasatinib. Mitochondrial dysfunction is an important component of apoptosis, and notably mitochondrial membrane potential is one of the most significant events (Ly et al, 2003). To explore the role of mitochondrial dysfunction in apoptosis induced by combination therapy of dasatinib and SN38, we applied flow cytometry with JC-1 staining to analyse mitochondrial dysfunction after $24 \mathrm{~h}$ treatment. As elucidated in Figures 3A and B, SN38 synergised with dasatinib gave rise to the decrease of mitochondrial membrane potential compared with single agent. The JC-1 green cells of the control, dasatinib, SN38 and combination groups in HepG-2 were 7.57, $11.77,20.83$ and $35.76 \%$, respectively, and those in BEL-7402 were $17.15,27.76,26.04$ and $70.88 \%$, respectively.

In addition, Bcl-2 family had an important role in regulating the mitochondrial pathway of apoptosis (Martinou and Youle, 2011). Bcl-2 was known to be an antiapoptotic protein and Bax was recognised as a pro-apoptotic protein (Brunelle and Letai, 2009). Inactivation of $\mathrm{Bcl}-2$ and activation of Bax were obviously detected in HCC cells treated with both dasatinib and SN38 (Figure 3C), and it was demonstrated that Bcl-2 family were able to activate downstream apoptosis pathways, such as caspase activation, apoptosis and cell death (Danial and Korsmeyer, 2004), which was consistent with our previous results. These data implicated that
A
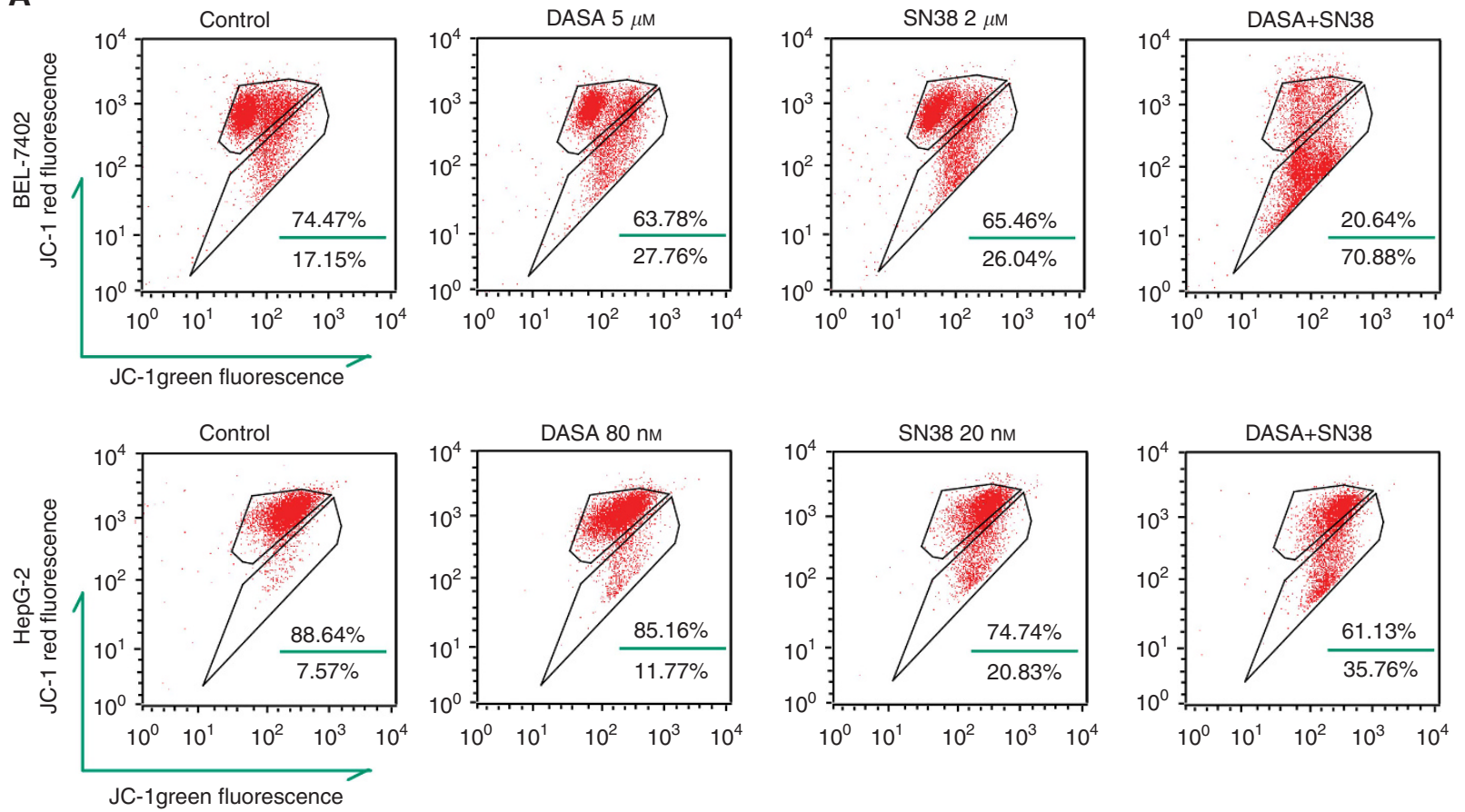

B

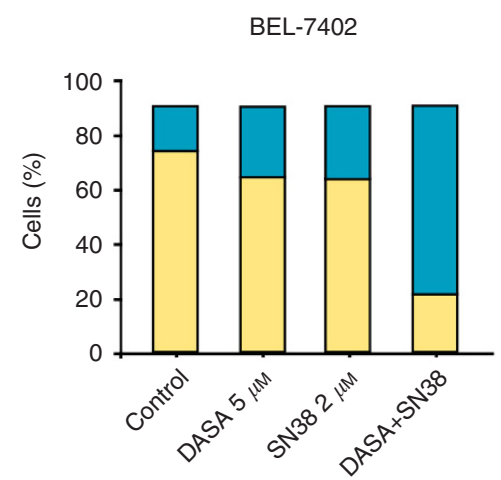

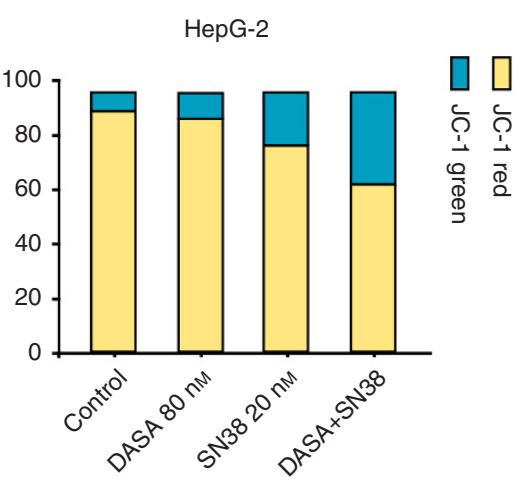
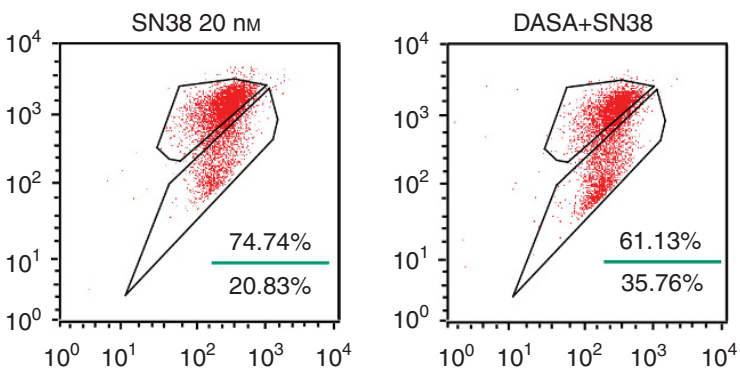

C

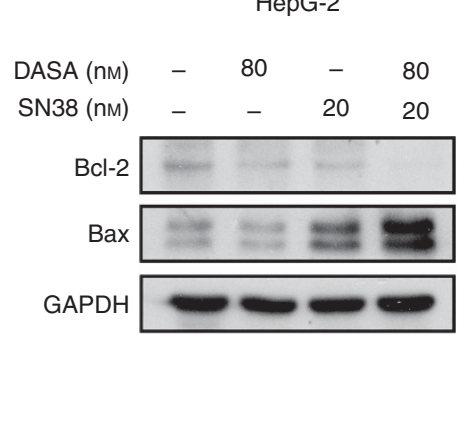

Figure 3. Mitochondrial dysfunction was involved in caspase-dependent apoptosis. (A) Flow cytometry with JC-1 staining was applied to analyse the loss of mitochondrial membrane potential in BEL-7402 and HepG-2 after $24 \mathrm{~h}$ treatment of dasatinib and/or SN38. (B) The rates of red to green of BEL-7402 and HepG-2 treated with dasatinib and/or SN38 are shown. (C) The effects of combination treatment of dasatinib and SN38 on the protein expression of $\mathrm{Bcl}-2$ and Bax were were analysed by western blotting. 
the combination therapy of dasatinib and SN38 induced mitochondrial related-apoptosis.

PLK1 is an important factor restricting antitumour effect of SN38 on HepG-2 cell line. As mentioned above, the anti-HCC efficacy of SN38 is limited due to dose-related side effects (O'Reilly et al, 2001). We noticed that PLK1 is one of the determinants of tumour cell sensitivity to SN38 (Zuco et al, 2015) and could promote cell survival via blocking mitochondrial dysfunction (Matsumoto et al, 2009). Assuming that PLK1 may have an important role in the limitation of SN38, we treated HepG-2 cells with SN38 at different concentrations and different periods of time. As a result, downregulation of PLK1 was observed and consistent with time or concentration gradients. However, the PLK1 inhibition induced by SN38 is not thorough under the concentrations and time periods of our experiments as shown in Figure 4A. At the same time, qRT-PCR results as shown in Figure $4 \mathrm{~B}$ indicated that downregulation of PLK1 triggered by SN38 was highly possible to be a result of transcription inhibition.

For validation of PLK1's role, we detected the relation between the inhibition rate of PLK1 and apoptosis in HepG-2 cells. As shown in Figure 4C, along with the downregulation of PLK1 by siRNA, cleaved PARP was increased obviously. And after we used different concentrations of PLK1 siRNAs to downregulate PLK1, as can be seen in Figure 4D, with more inhibition of PLK1, upraised cleaved PARP was detected by western blotting. Furthermore, when we silenced PLK1 in HepG-2 cells, the cleaved PARP level was further arisen (Figure 4E), and when PLK1 was overexpressed, the SN-38-induced cleaved PARP level was recovered (Figure 4F). These data implicated that PLK1 had a part in the limitation of SN38 and thoroughly downregulation of PLK1 might be a way to reinforce the efficacy of SN38 on HepG-2 cells.

PLK1 downregulation is a key segment of combination therapyinduced apoptosis in HepG-2 cells and was achieved by the inhibition of protein synthesis. As the role of PLK1 in SN38 sensitivity has been confirmed, we assumed that accelerated PLK1 downregulation by dasatinib may be the precise mechanism of the syngestic anticancer activity. After treatment by dasatinib in different concentrations and different periods of time, downregulation of PLK1 was observed and consistent with time or concentration gradients as shown in Figure 5A. Compared with dasatinib or SN38 monotherapy, combination treatment was able to reduce the level of PLK1 more thoroughly as shown in Figure 5B. These data indicated that dasatinib was able to downregulate PLK1 level in HepG-2 cells and led to synergistic effects. This was confirmed by PLK1 overexpression, which showed that, after PLK1 was overexpressed, the arisen cleaved PARP level and cell death induced by combination of dasatinib and SN38 was decreased (Figures 5C and D).

To further study how dasatinib enhanced PLK1 inhibition, we incubated HepG-2 cells with lysosomal inhibitors chloroquine $(\mathrm{CQ}, 10 \mu \mathrm{M})$ and proteasome inhibitor MG132 $(2 \mu \mathrm{M})$ before combination treatment of SN38 (20 nM) and dasatinib (80 nM). Both CQ and MG132 had no effect on combination treatmentinduced suppression of PLK1, which denoted that degradation pathways were not involved in this combination therapy-induced PLK1 decrease (Figure 5E). Notably, the level of p-4EBP1 was observed to be reduced by combination treatment (Figure 5B), which implicated that the enhanced reduction of PLK1 might be a result of protein synthesis inhibition. For validation, CHX, an inhibitor of protein synthesis, was applied to prevent synthesis of PLK1 in HepG-2 cells as previously described (Liu et al, 2014). We determined that combination treatment was unable to further reduce the level of PLK1 after CHX pretreatment, as shown in Figure 5F. In sum, the combination treatment of SN38 and dasatinib shows anti-HCC effect chiefly through downregulating PLK1 protein levels in the way of inhibiting protein synthesis.

The synergistic antitumour effect of dasatinib and irinotecan in HepG-2 xenografts. To further assess the synergistic antitumour effect of two agents, we detected the in vivo efficacy in HepG-2 xenograft nude mice models ( $n=6$ per group). Dasatinib was
A

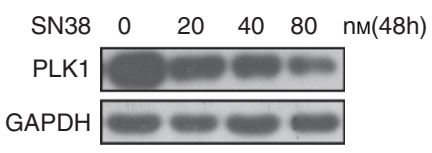

SN38 $20 \mathrm{~nm}$ Oh $12 \mathrm{~h} \quad 24 \mathrm{~h} 48 \mathrm{~h}$

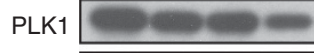

GAPDH

B

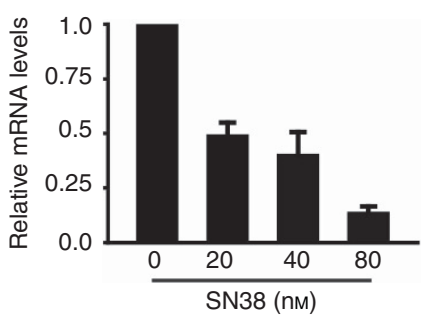

C

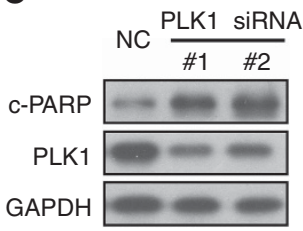

E

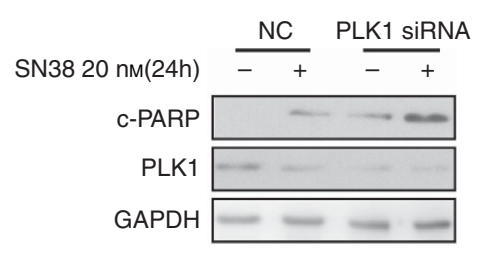

$\mathbf{F}$

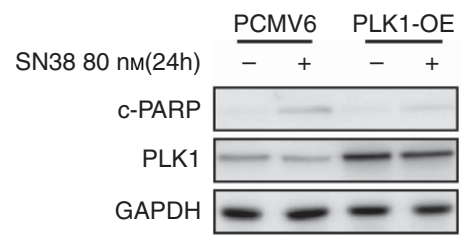

Figure 4. PLK1 is an important factor restricting antitumour effect of SN38 on HepG-2 cell line. (A) SN38 could downregulate PLK1 in both doseand time-dependent manner. The downregulation was not thorough. (B) Results of qRT-PCR showed that SN38 reduced PLK1 mRNA level in HepG-2 cells. (C) PLK1 knockdown was achieved by transfection with different PLK1 siRNAs, and western blotting with specific antibodies to PLK1 and cleaved PARP were used to confirm the effects of PLK1 knockdown. (D) Different concentrations of PLK1 siRNAs were used to downregulate PLK1, and the protein levels of PLK1 and cleaved PARP were detected by western blotting. (E) Twenty-four hours after silence of PLK1 by siRNA, HepG-2 cells were treated with $20 \mathrm{~nm} \mathrm{SN38} \mathrm{for} 24 \mathrm{~h}$. PLK1 siRNA was able to enhance the uprising of c-PARP level induced by SN38 treatment. (F) Twenty-four hours after transfection with PLK1 expression plasmid or empty vector (PCMV6), HepG-2 cells were treated with 80 nM SN38 for 24h. Overexpression of PLK1 by plasmid was able to reverse the arisen c-PARP level induced by SN38 in vector (PCMV6) group. 
A

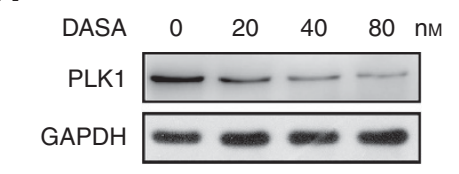

DASA $80 \mathrm{~nm} \quad 0 \quad 12 \quad 24 \quad 48 \quad \mathrm{~h}$

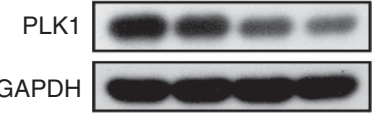

B

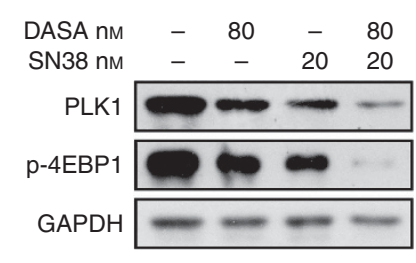

C

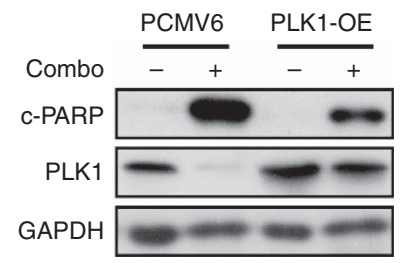

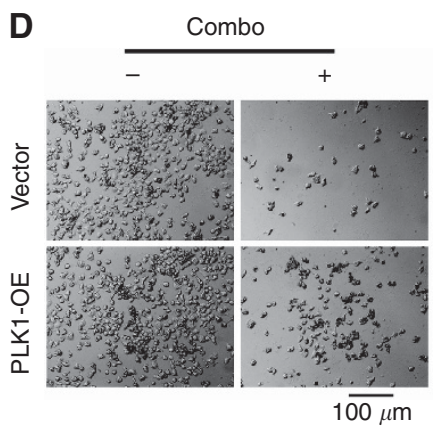

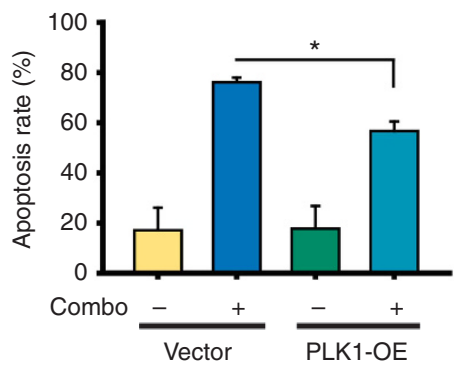

E
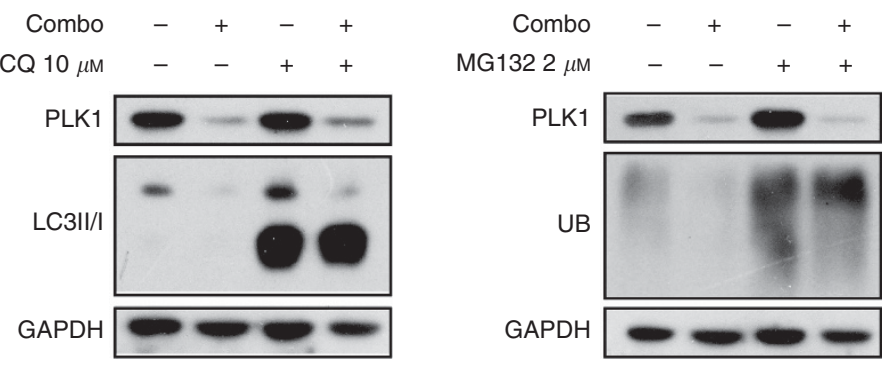

$\mathbf{F}$

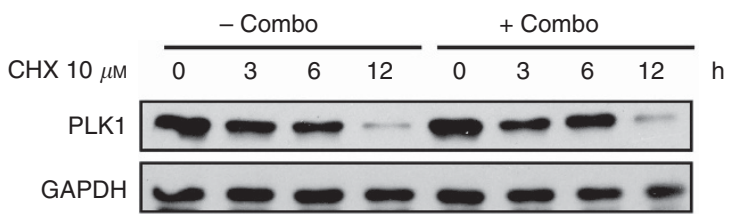

Figure 5. Inhibition of PLK1 was the key factor of synergistic therapy-induced apoptosis and was achieved by the inhibition of protein synthesis. (A) Dasatinib inhibited PLK1 dose and time dependently. (B) The effects of combination treatment of dasatinib and SN38 on the protein expression of PLK1 and p-4EBP1 were analysed by western blotting. Both PLK1 and p-4EBP1 level were reduced. (C, D) Twenty-four hours after transfection with PLK1 expression plasmid or empty vector (PCMV6), HCC cells were treated with both $20 \mathrm{~nm}$ SN38 and $80 \mathrm{~nm}$ dasatinib for $48 \mathrm{~h}$, and the inhibition of proliferation was observed by optical microscopy, while cleaved PARP and PLK1 were assessed by western blotting. Both cell death and c-PARP level were partially reversed. The reversed cell death was also confirmed by PI staining and flow cytometry. (E) HepG-2 cells were pretreated with CQ $(10 \mu \mathrm{M})$ or MG132 $(2 \mu \mathrm{M})$ for $2 \mathrm{~h}$ and then treated with both SN38 $(20 \mathrm{~nm})$ and dasatinib $(80 \mathrm{~nm})$ for $24 \mathrm{~h}$, and the protein levels of PLK1 were measured by western blotting. (F) HepG2 cells were pretreated with both SN38 (20 nM) and dasatinib ( $80 \mathrm{nm)} \mathrm{or} \mathrm{not} \mathrm{before}$ exposing to $\mathrm{CHX}(10 \mu \mathrm{m})$ for different times, and PLK1 protein levels were detected by western blotting.

administered i.g. at $100 \mathrm{mg} \mathrm{kg}^{-1}$ once daily, and the i.p. administration of irinotecan at a dose of $1 \mathrm{mg} \mathrm{kg}^{-1}$ every 2 days was conducted. As shown in Figure 6A, neither dasatinib $(0.9077 \pm 0.0468 \mathrm{~g})$ nor irinotecan $(0.9757 \pm 0.1202 \mathrm{~g})$ produced significant difference on the inhibition of tumour growth compared with that of the control group $(1.0520 \pm 0.1196 \mathrm{~g})$, while the combination of dasatinib and irinotecan robustly suppressed tumour growth and restrained tumour weight $(0.5577 \pm 0.1101 \mathrm{~g})$. As predicted, tumour volume and relative tumour volume were remarkably inhibited in the combination treatment group, showing a similar trend to the weight of tumour (Figure 6B). At the same time, no animal death or significant body weight change was observed in all groups during the experiments, which suggested that the combination therapy was well tolerated (Figure 6C).

In order to determine whether the synergistic antitumour effect was conducted by decreasing PLK1 and increasing tumour apoptosis in vivo, we detected the expression of apoptosis-related proteins and PLK1 in tumour tissues from xenograft mice. Consistent with our previous in vitro results, in vivo data showed that the cleavage of PARP and caspase- 3 increased greatly in the combination treatment group, implying the activation of caspase cascades. Besides, the inhibition of PLK1 caused by irinotecan was reinforced by dasatinib (Figure 6D). In sum, these results demonstrated that dasatinib induced inhibition of PLK1 and enhanced the efficacy of irinotecan/SN38 in vivo, which consequently reduced the dose of $\mathrm{SN} 38$ to a relatively safe level in xenograft model.

\section{DISCUSSION}

HCC has a long history, and despite all the medical efforts, it still remains essentially incurable especially for advanced cases (Schutte et al, 2014; Tejeda-Maldonado et al, 2015). One of the most important reasons of this unfavourable situation is the lack of efficient anti-HCC agents. In this work, we demonstrated that combination treatment of dasatinib and irinotecan/SN38 led to augmented antitumour effect on HCC both in vitro and in vivo. This effect relies on the inhibition of PLK1, which is enhanced by dasatinib through its effect on the protein synthesis progress.

PLK1 is a member of a serine/threonine kinases (PLK1-4) family and highly associated with G2 checkpoint (Lens et al, 2010; Liang et al, 2014) and is widely believed to have an important role 
A

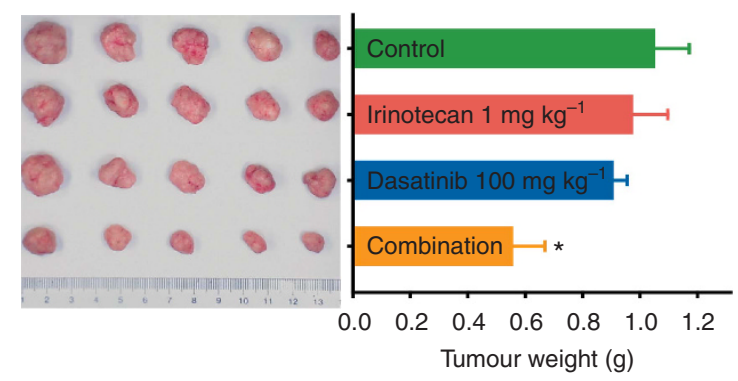

B

\begin{tabular}{ccccc}
\hline & Control & $\begin{array}{c}\text { Irinotecan } \\
\left(1 \mathrm{mg} \mathrm{kg}^{-1}\right)\end{array}$ & $\begin{array}{c}\text { Dasatinib } \\
\left(100 \mathrm{mg} \mathrm{kg}^{-1}\right)\end{array}$ & $\begin{array}{c}\text { Irinotecan } \\
\text { +Dasatinib }\end{array}$ \\
\hline Tumour volume $\left(\mathrm{mm}^{3}\right)$ & $1294.97 \pm 179.89$ & $1156.40 \pm 148.35$ & $1106.4 \pm 56.5$ & $651.72 \pm 106.95$ \\
Tumour inhibiting rates-TV & $/$ & $10.70 \%$ & $14.56 \%$ & $49.67 \% \%^{*}$ \\
Relative tumour volume & $8.08 \pm 0.66$ & $6.99 \pm 0.77$ & $6.91 \pm 0.58$ & $3.95 \pm 0.26$ \\
Relative tumour inhibiting rates-RTV & $/$ & $13.57 \%$ & $14.57 \%$ & $51.17 \% \%^{*}$ \\
\hline
\end{tabular}

C

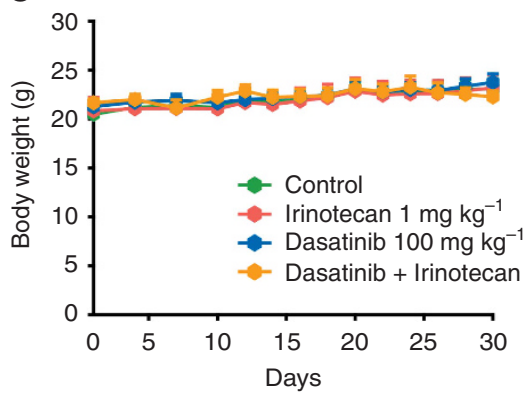

D Dasatinib mg kg${ }^{-1}-100 \quad-100$

Irinotecan $\mathrm{mg} \mathrm{kg}^{-1} \quad-\quad-\quad \begin{array}{lll}1 & 1\end{array}$

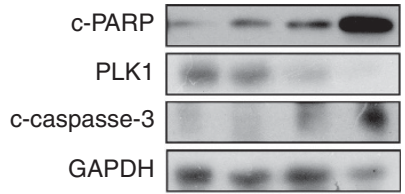

Figure 6. Combination treatment of dasatinib and irinotecan showed synergistic antitumour efficacy in HepG-2 xenografts. Nude mice transplanted with HepG-2 xenografts were treated with dasatinib $\left(100 \mathrm{mg} \mathrm{kg}^{-1}\right.$, i.g.), irinotecan $\left(1 \mathrm{mg} \mathrm{kg}^{-1}\right.$, i.p.), combination or vehicle for 30 days $\left(n=6\right.$ per group). (A) Morphology of tumours are shown and the weight of tumour were described as the mean \pm s.e.m. ${ }^{\star}$ Compared with the monotherapy groups, $P<0.05$. (B) Tumour volume and relative tumour volume are shown and described as the mean \pm s.e.m., and their corresponding tumour inhibition rates at day 30 are also shown in the table. ${ }^{\star}$ Compared with the monotherapy groups, $P<0.05$. (C) Average body weights are shown and expressed as the mean \pm s.e.m. (D) Western blotting showed cleaved PARP, cleaved caspase-3, PLK1 and GAPDH from proteins extracted from tumour tissues of different groups of mice.

in cell proliferation (Raab et al, 2015). The function of PLK1 could be related to various behaviours of tumour cells (Strebhardt and Ullrich, 2006) and thus the overexpression of PLK1 in HCC is often related to poor prognosis (He et al, 2009). Based on the importance of PLK1, it is supposed to be a desirable target. In fact, PLK1 has proved to strongly influence SN38 sensitivity of tumour cells (Zuco et al, 2015). Our data showed that SN38 did decrease the level of PLK1 in HCC cells but not thoroughly. The decrease was dependent on the concentration of SN38, which means if PLK1 could be inhibited by other methods, the concentration of SN38 could be lower to show effect. This might be a way to deal with the dose-dependent toxicity of irinotecan, which limits its effect on HCC.

Dasatinib is a relatively well-studied antitumour agent. Its targets include a number of different molecules and pathways. PLK1 is not one of them, according to current perception. In fact, the relationship of PLK1 and dasatinib is almost unreported. However, the mechanism by which dasatinib influences certain downstream molecules remains heuristic. The way dasatinib acts on receptor tyrosine kinases such as c-kit and PDGFR is to block their kinase activity (Karaman et al, 2008). Except decreasing activity, dasatinib can also downregulate the amount of some targets - often downstream proteins. Dasatinib is proved to induce apoptosis and cell cycle arrest in some kinds of tumour cells (Johnson et al, 2005) through the regulation of effectors molecules, such as p21, cyclin D1, CDK2 and CDK8. Among these, CDK2 and CDK8 are reported to be suppressed through gene modulation (Nunoda et al, 2007). Which indicates that dasatinib is able to inhibit targets by downregulating their synthesis level, which is determined by gene expression.

According to the pattern in which dasatinib acts on CDK2 and CDK8 and the role of PLK1 in the antitumour effect of SN38, whether dasatinib could influence PLK1 by interfering with its synthesis is worth considering. This was confirmed by our data which showed that dasatinib could enhance the PLK1 inhibition of SN38 due to the blockage of protein synthesis. It is the first time that dasatinib is proved to have influence on PLK1. We also ruled out the possibility of dasatinib inhibiting PLK1 through protein degradation progress. The exact mechanism is likely to be a new direction of further study.

Of note, it is highly possible that the way in which dasatinib inhibits PLK1 differs from those of existing PLK1 inhibitors. Currently, there are a number of PLK1 inhibitors undergoing clinical trials such as Volasertib (Van den Bossche et al, 2016) and Rigosertib (Bowles et al, 2014; Garcia-Manero et al, 2016; O’Neil et al, 2016), and whether they are ATP-binding competitive or not, these PLK1 inhibitors target on PLK1 kinase activity (Schoffski, 2009) instead of reducing its amount. This feature might limit the application range of the existing PLK1 inhibitors to some extent as not all tumours with high level of PLK1 expression necessarily show arisen kinase activity. In fact, the target diseases of current clinical trials of Volasertib and Rigosertib are quite restricted (Schoffski, 2009) compared with the wide range of PLK1 overexpression (Strebhardt, 2010), and HCC is not included in the trials. Under this circumstance, the finding that dasatinib is able to inhibit protein synthesis of PLK1 presents a new method targeting on PLK1 and might have better universality especially on HCC. Apart from the restricted application range, existing PLK1 inhibitors also have to face potential problems about drug resistance. It is believed that one of the most common reasons why acquired resistance of inhibitors occurs is point mutation of the kinase domain (Daver et al, 2015). Which means, compared with PLK1 inhibitors targeting on kinase activity, dasatinib will suffer less risk of developing acquired resistance, as its mechanism depends on protein synthesis instead of gene level or kinase structure.

Given to the close correlation of PLK1 and tumour cell proliferation, enhanced inhibition of PLK1 is naturally related to increased apoptosis (Bucur et al, 2013). It was reported that mitochondria dysfunction could be involved in the progress from PLK1 inhibition to apoptosis (Matsumoto et al, 2009). Consistent with these previous works, our study also revealed that the apoptosis rate of HCC cells was elevated as a result of PLK1 inhibition and mitochondria dysfunction, and a synergistic antitumour effect could be observed both in vitro and in vivo. 
In conclusion, PLK1 might be a potential target of dasatinib and the combination treatment of dasatinib and irinotecan/SN38 led to augmented inhibition of PLK1, which subsequently enhanced irinotecan/SN38-induced mitochondrial pathway of apoptosis. This study presented a promising synergistic antitumour efficacy of a traditional cytotoxic drug and a TKI for HCC and may hopefully inspire novel therapies for the disease.

\section{ACKNOWLEDGEMENTS}

This work was supported by the National Natural Science Foundation of China (No. 81473288), Zhejiang Province Joint Construction Project (No. WSK2014-2-007) and Fundamental Research Funds for the Central Universities.

\section{CONFLICT OF INTEREST}

The authors declare no conflict of interest.

\section{REFERENCES}

Barr FA, Sillje HH, Nigg EA (2004) Polo-like kinases and the orchestration of cell division. Nat Rev Mol Cell Biol 5(6): 429-440.

Bowles DW, Diamond JR, Lam ET, Weekes CD, Astling DP, Anderson RT, Leong S, Gore L, Varella-Garcia M, Vogler BW, Keysar SB, Freas E, Aisner DL, Ren C, Tan AC, Wilhelm F, Maniar M, Eckhardt SG, Messersmith WA, Jimeno A (2014) Phase I study of oral rigosertib (ON 01910.Na), a dual inhibitor of the PI3K and Plk1 pathways, in adult patients with advanced solid malignancies. Clin Cancer Res 20(6): $1656-1665$.

Bruix J, Sherman M, American Association for the Study of Liver Disease (2011) Management of hepatocellular carcinoma: an update. Hepatology 53(3): 1020-1022.

Brunelle JK, Letai A (2009) Control of mitochondrial apoptosis by the Bcl-2 family. J Cell Sci 122(Pt 4): 437-441.

Bucur O, Stancu AL, Goganau I, Petrescu SM, Pennarun B, Bertomeu T, Dewar R, Khosravi-Far R (2013) Combination of bortezomib and mitotic inhibitors down-modulate $\mathrm{Bcr}-\mathrm{Abl}$ and efficiently eliminates tyrosinekinase inhibitor sensitive and resistant Bcr-Abl-positive leukemic cells. PLoS One 8(10): e77390.

Bupathi M, Kaseb A, Meric-Bernstam F, Naing A (2015) Hepatocellular carcinoma: Where there is unmet need. Mol Oncol 9(8): 1501-1509.

Chabalier-Taste C, Brichese L, Racca C, Canitrot Y, Calsou P, Larminat F (2016) Polo-like kinase 1 mediates BRCA1 phosphorylation and recruitment at DNA double-strand breaks. Oncotarget 7(3): 2269-2283.

Chou TC (2010) Drug combination studies and their synergy quantification using the Chou-Talalay method. Cancer Res 70(2): 440-446.

Danial NN, Korsmeyer SJ (2004) Cell death: critical control points. Cell 116(2): 205-219.

Daver N, Cortes J, Ravandi F, Patel KP, Burger JA, Konopleva M, Kantarjian H (2015) Secondary mutations as mediators of resistance to targeted therapy in leukemia. Blood 125(21): 3236-3245.

Garcia-Manero G, Fenaux P, Al-Kali A, Baer MR, Sekeres MA, Roboz GJ, Gaidano G, Scott BL, Greenberg P, Platzbecker U, Steensma DP, Kambhampati S, Kreuzer KA, Godley LA, Atallah E, Collins Jr R, Kantarjian H, Jabbour E, Wilhelm FE, Azarnia N, Silverman LR, ONTIME study investigators (2016) Rigosertib versus best supportive care for patients with high-risk myelodysplastic syndromes after failure of hypomethylating drugs (ONTIME): a randomised, controlled, phase 3 trial. Lancet Oncol 17(4): 496-508.

He ZL, Zheng H, Lin H, Miao XY, Zhong DW (2009) Overexpression of polo-like kinase1 predicts a poor prognosis in hepatocellular carcinoma patients. World J Gastroenterol 15(33): 4177-4182.

Jin Z, El-Deiry WS (2005) Overview of cell death signaling pathways. Cancer Biol Ther 4(2): 139-163.

Johnson FM, Saigal B, Talpaz M, Donato NJ (2005) Dasatinib (BMS-354825) tyrosine kinase inhibitor suppresses invasion and induces cell cycle arrest and apoptosis of head and neck squamous cell carcinoma and non-small cell lung cancer cells. Clin Cancer Res 11 (19 Pt 1): 6924-6932.

Karaman MW, Herrgard S, Treiber DK, Gallant P, Atteridge CE, Campbell BT, Chan KW, Ciceri P, Davis MI, Edeen PT, Faraoni R, Floyd M, Hunt JP, Lockhart DJ, Milanov ZV, Morrison MJ, Pallares G, Patel HK, Pritchard S, Wodicka LM, Zarrinkar PP (2008) A quantitative analysis of kinase inhibitor selectivity. Nat Biotechnol 26(1): 127-132.

Lens SM, Voest EE, Medema RH (2010) Shared and separate functions of polo-like kinases and aurora kinases in cancer. Nat Rev Cancer 10(12): 825-841.

Liang H, Esposito A, De S, Ber S, Collin P, Surana U, Venkitaraman AR (2014) Homeostatic control of polo-like kinase-1 engenders non-genetic heterogeneity in G2 checkpoint fidelity and timing. Nat Commun 5: 4048.

Liu XW, Cai TY, Zhu H, Cao J, Su Y, Hu YZ, He QJ, Yang B (2014) Q6, a novel hypoxia-targeted drug, regulates hypoxia-inducible factor signaling via an autophagy-dependent mechanism in hepatocellular carcinoma. Autophagy 10(1): 111-122.

Ly JD, Grubb DR, Lawen A (2003) The mitochondrial membrane potential (deltapsi(m)) in apoptosis; an update. Apoptosis 8(2): 115-128.

Martinou JC, Youle RJ (2011) Mitochondria in apoptosis: Bcl-2 family members and mitochondrial dynamics. Dev Cell 21(1): 92-101.

Matsumoto T, Wang PY, Ma W, Sung HJ, Matoba S, Hwang PM (2009) Polo-like kinases mediate cell survival in mitochondrial dysfunction. Proc Natl Acad Sci USA 106(34): 14542-14546.

Mayer EL, Baurain JF, Sparano J, Strauss L, Campone M, Fumoleau P, Rugo H, Awada A, Sy O, Llombart-Cussac A (2011) A phase 2 trial of dasatinib in patients with advanced HER2-positive and/or hormone receptor-positive breast cancer. Clin Cancer Res 17(21): 6897-6904.

Milano V, Piao Y, LaFortune T, de Groot J (2009) Dasatinib-induced autophagy is enhanced in combination with temozolomide in glioma. Mol Cancer Ther 8(2): 394-406.

Montero JC, Seoane S, Ocana A, Pandiella A (2011) Inhibition of SRC family kinases and receptor tyrosine kinases by dasatinib: possible combinations in solid tumors. Clin Cancer Res 17(17): 5546-5552.

Njei B, Rotman Y, Ditah I, Lim JK (2015) Emerging trends in hepatocellular carcinoma incidence and mortality. Hepatology 61(1): 191-199.

Nunoda K, Tauchi T, Takaku T, Okabe S, Akahane D, Sashida G, Ohyashiki JH, Ohyashiki K (2007) Identification and functional signature of genes regulated by structurally different ABL kinase inhibitors. Oncogene 26(28): 4179-4188.

O’Neil BH, Scott AJ, Ma WW, Cohen SJ, Aisner DL, Menter AR, Tejani MA, Cho JK, Granfortuna J, Coveler AL, Olowokure OO, Baranda JC, Cusnir M, Phillip P, Boles J, Nazemzadeh R, Rarick M, Cohen DJ, Radford J, Fehrenbacher L, Bajaj R, Bathini V, Fanta P, Berlin J, McRee AJ, Maguire R, Wilhelm F, Maniar M, Jimeno A, Gomes CL, Messersmith WA (2016) A phase II/III randomized study to compare the efficacy and safety of rigosertib plus gemcitabine versus gemcitabine alone in patients with previously untreated metastatic pancreatic cancer. Ann Oncol 27(6): 1180.

O'Reilly EM, Stuart KE, Sanz-Altamira PM, Schwartz GK, Steger CM, Raeburn L, Kemeny NE, Kelsen DP, Saltz LB (2001) A phase II study of irinotecan in patients with advanced hepatocellular carcinoma. Cancer 91(1): 101-105.

Ottolino-Perry K, Acuna SA, Angarita FA, Sellers C, Zerhouni S, Tang N, McCart JA (2015) Oncolytic vaccinia virus synergizes with irinotecan in colorectal cancer. Mol Oncol 9(8): 1539-1552.

Raab M, Kramer A, Hehlgans S, Sanhaji M, Kurunci-Csacsko E, Dotsch C, Bug G, Ottmann O, Becker S, Pachl F, Kuster B, Strebhardt K (2015) Mitotic arrest and slippage induced by pharmacological inhibition of Pololike kinase 1. Mol Oncol 9(1): 140-154.

Schoffski P (2009) Polo-like kinase (PLK) inhibitors in preclinical and early clinical development in oncology. Oncologist 14(6): 559-570.

Schutte K, Schulz C, Malfertheiner P (2014) Hepatocellular carcinoma: current concepts in diagnosis, staging and treatment. Gastrointest Tumors 1(2): 84-92.

Secord AA, Teoh DK, Barry WT, Yu M, Broadwater G, Havrilesky LJ, Lee PS, Berchuck A, Lancaster J, Wenham RM (2012) A phase I trial of dasatinib, an SRC-family kinase inhibitor, in combination with paclitaxel and carboplatin in patients with advanced or recurrent ovarian cancer. Clin Cancer Res 18(19): 5489-5498.

Somlo G, Atzori F, Strauss LC, Geese WJ, Specht JM, Gradishar WJ, Rybicki A, Sy O, Vahdat LT, Cortes J (2013) Dasatinib plus capecitabine for advanced breast cancer: safety and efficacy in phase I study CA180004. Clin Cancer Res 19(7): 1884-1893. 
Strebhardt K (2010) Multifaceted polo-like kinases: drug targets and antitargets for cancer therapy. Nat Rev Drug Discov 9(8): 643-660.

Strebhardt K, Ullrich A (2006) Targeting polo-like kinase 1 for cancer therapy. Nat Rev Cancer 6(4): 321-330.

Takeba Y, Kumai T, Matsumoto N, Nakaya S, Tsuzuki Y, Yanagida Y, Kobayashi S (2007) Irinotecan activates p53 with its active metabolite, resulting in human hepatocellular carcinoma apoptosis. J Pharmacol Sci 104(3): 232-242.

Talpaz M, Shah NP, Kantarjian H, Donato N, Nicoll J, Paquette R, Cortes J, O’Brien S, Nicaise C, Bleickardt E, Blackwood-Chirchir MA, Iyer V, Chen TT, Huang F, Decillis AP, Sawyers CL (2006) Dasatinib in imatinibresistant Philadelphia chromosome-positive leukemias. $N$ Engl J Med 354(24): 2531-2541.

Tejeda-Maldonado J, Garcia-Juarez I, Aguirre-Valadez J, Gonzalez-Aguirre A, Vilatoba-Chapa M, Armengol-Alonso A, Escobar-Penagos F, Torre A, Sanchez-Avila JF, Carrillo-Perez DL (2015) Diagnosis and treatment of hepatocellular carcinoma: an update. World J Hepatol 7(3): 362-376.

Torre LA, Bray F, Siegel RL, Ferlay J, Lortet-Tieulent J, Jemal A (2015) Global cancer statistics, 2012. CA Cancer J Clin 65(2): 87-108.

Van den Bossche J, Lardon F, Deschoolmeester V, De Pauw I, Vermorken JB, Specenier P, Pauwels P, Peeters M, Wouters A (2016) Spotlight on volasertib: preclinical and clinical evaluation of a promising Plk1 inhibitor. Med Res Rev 36(4): 749-786.

Vichai V, Kirtikara K (2006) Sulforhodamine B colorimetric assay for cytotoxicity screening. Nat Protoc 1(3): 1112-1116.

Viktorsson K, Shah CH, Juntti T, Haag P, Zielinska-Chomej K, Sierakowiak A, Holmsten K, Tu J, Spira J, Kanter L, Lewensohn R, Ullen A (2016) Melphalan-flufenamide is cytotoxic and potentiates treatment with chemotherapy and the Src inhibitor dasatinib in urothelial carcinoma. Mol Oncol 10(5): 719-734.

Zuco V, De Cesare M, Zaffaroni N, Lanzi C, Cassinelli G (2015) PLK1 is a critical determinant of tumor cell sensitivity to CPT11 and its inhibition enhances the drug antitumor efficacy in squamous cell carcinoma models sensitive and resistant to camptothecins. Oncotarget 6(11): 8736-8749.

(c) (1) (2) (2) This work is licensed under the Creative Commons BY ${ }_{\text {NC }}$ SA Attribution-Non-Commercial-Share Alike 4.0 International License. To view a copy of this license, visit http:// creativecommons.org/licenses/by-nc-sa/4.0/

(C) The Author(s) named above 2017

Supplementary Information accompanies this paper on British Journal of Cancer website (http://www.nature.com/bjc) 